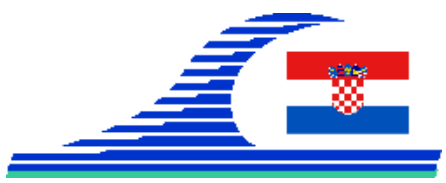

\title{
Coastal evolution monitoring by Earth Observation
}

\author{
Antoine MANGIN ${ }^{1,2}$, Amine Mohammed TAJI ${ }^{2}$
}

1. ACRI-HE Sophia, 260 route du pin Montard, BP 234, 06904 Sophia-Antipolis, France.

antoine.mangin@acri-he.fr,

2. ACRI-EC, 209 rue Mustapha El Maâni, Etage 6, Appart. 10, Casablanca, Morocco.

amine.taji@acriec.ma

\begin{abstract}
:
After a severe flood that has occurred in South of Morocco last year (October), a monitoring system based on the use of Satellite data from EU-Copernicus program, has been set up. It allows following coastline evolution and can be used for further estimation of sediment transport rates.
\end{abstract}

\section{Keywords:}

Sedimentary processes, Longshore sediment transport, Copernicus, Sentinel 2, Coastline evolution

\section{Introduction}

An exceptional flood has occurred at the end of October 2016 in the South of Morocco (Laayoune). The river (oued) peak flow has been estimated at 8 times the value that was foreseen for a 50-year return period flood. A dam was built in 1992 at $12 \mathrm{~km}$ from the shoreline to serve as reservoir for the water needs of town of Laayoune (about 100.000 inhabitants) and to reinject water into the aquifer to limit intrusion of salt waters. The dam as well as main infrastructures downstream, have been destroyed by the flood. A coastal infrastructure was under study in the axis of the flood, and the construction site and existing coastal road has been significantly impacted. After the flood an expertise has been ordered to ACRI, to make the analysis of the sedimentary situation, to check whether the coastline is back to a "stable' state and to investigate remediation actions to re-start the construction site. We present here the use of Earth Observation from Satellite mission which has been used to monitor the coastline evolution and the sediment flux since the flood event (one year ago)

\section{Materials and methods}

Few years ago, the European Commission has launched the Copernicus program (in continuation of GMES activities) in order to ensure a European consistent and 
Mediterranean rocky coasts:

Features, processes, evolution and problems

sustainable means of observations of the environment. In situ data and satellite (Earth Observation) information of all nature are available for air, land, risk, coastal and marine monitoring. The Copernicus program is intended to last for at least twenty years so environmental monitoring techniques making use of these data can be set up as a sustainable tool for the next decades. To what concerns satellite data, three types of missions have already been launched (Sentinel-1 for radar acquisition, Sentinel-2 for Very High resolution imagery (10m resolution in the visible bands) and Sentinel-3 for Medium resolution imagery (300m resolution in visible and thermal bands). All data is available can be downloaded free of charge. In this study we present the use of Sentinel2 data for monitoring the coastline evolution and stabilisation. For each available image (cloud-free) the coastline is extracted from the radiometry.

\section{Results and discussion}

We present on the following image (figure 1) the coastline evolution for the last months. The sea level (expressed from the nautical chart datum) is also indicated. From these information (a little less than 15 Sentinel-2 images have been used), an evolution of the coastline and of the transported volume of sediment have been assessed.

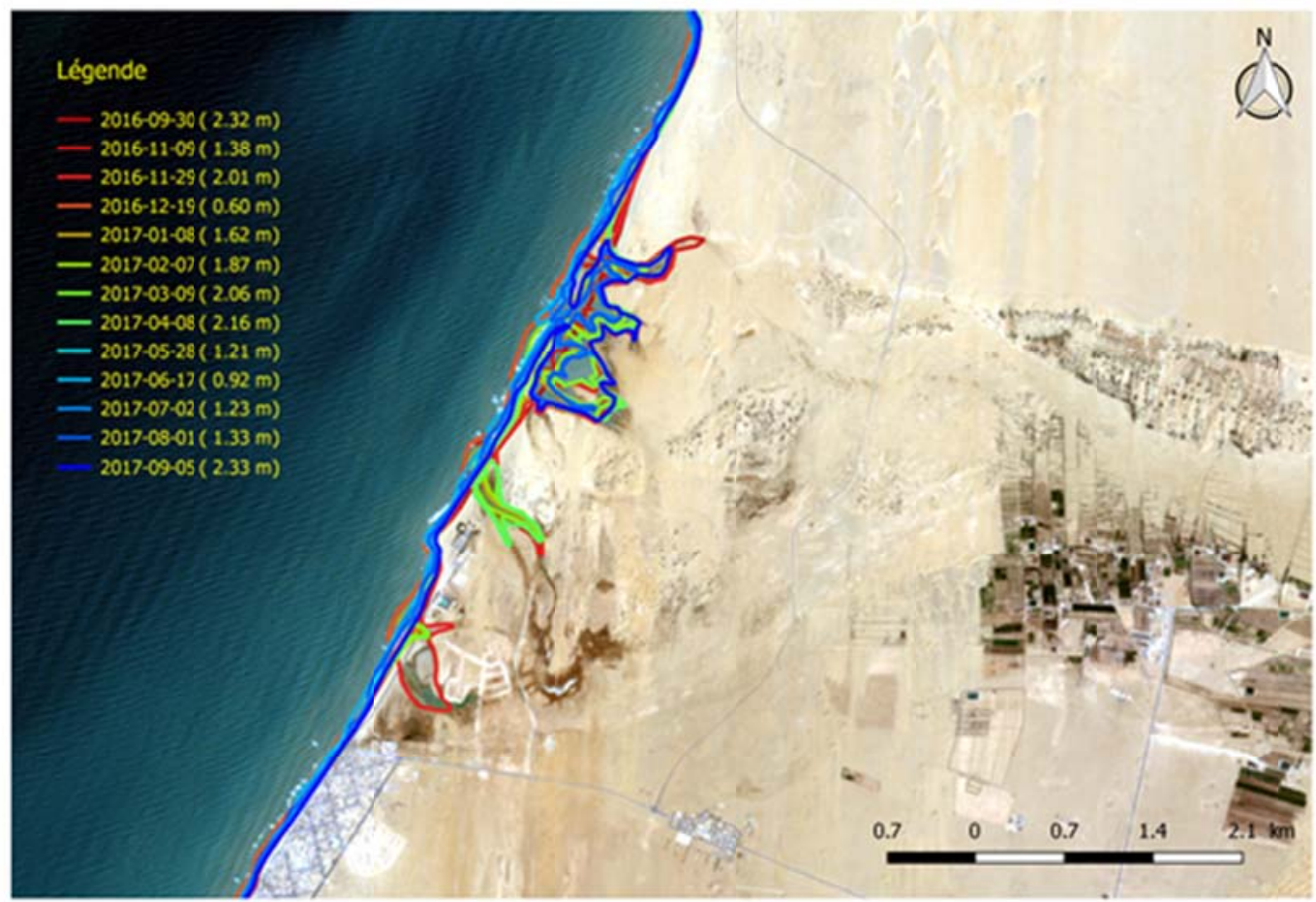

Figure 1. Evolution of coastline, Laayoune, from 2016, September to 2017, September. 
Mediterranean rocky coasts:

Features, processes, evolution and problems

\section{Conclusions}

The method that has been used here for coastline evolution is not very innovative in itself (this has been largely used in the past but with one or a couple of images). The novelty comes from the fact that a free flux of EO data is now available from Copernicus program that open the door to enough information to do dynamic studies and bring information on the estimation of longshore sedimentary transport (which, although being very sensitive information, is always difficult to validate). 
Mediterranean rocky coasts:

Features, processes, evolution and problems 